\title{
La publicación de artículos en las áreas de ciencias sociales y humanidades analizada a partir de los sistemas de información sobre investigación: el caso de las Universidades de Barcelona y Girona
}

\author{
Jordi Ardanuy* \\ * Departamento de Biblioteconomía y Documentación. Universitat de Barcelona \\ Correo-e: Jordi_ardanuy@ub.edu
}

Recibido: 12-03-2013; 2a version: 10-06-2013; Aceptado: 02-07-2013.

Cómo citar este artículo/Citation: Ardanuy, J. (2014). La publicación de artículos en las áreas de ciencias sociales y humanidades analizada a partir de los sistemas de información sobre investigación: el caso de las Universidades de Barcelona y Girona. Revista Española de Documentación Científica, 37(1):e035. doi: http://dx.doi.org/10.3989/redc.2014.1.1074

Resumen: Los sistemas de información sobre investigación y actividad científica de las universidades permiten complementar los datos que ofrecen las bases de datos multidisciplinares internacionales tales como WoS o Scopus, especialmente en disciplinas cuya cobertura no es suficientemente exhaustiva. En el presente estudio se analizan algunas características sobre los artículos y revistas publicados en las áreas de Ciencias Sociales y Humanidades por profesores e investigadores de dos universidades catalanas. Los datos han sido extraídos a partir de bases de datos que permiten el acceso libre a los registros de la producción de ambas instituciones académicas. Se concluye la importancia del acceso abierto a este tipo de registros además de la existencia de diferencias entre las áreas de conocimiento en aspectos tales como calidad, nivel de colaboración, porcentaje de divulgación, idioma de publicación, aunque existe una tendencia general a un mayor uso relativo de la lengua inglesa.

Palabras clave: Sistemas de información sobre la actividad científica; universidades; producción científica; Humanidades y Ciencias Sociales; Cataluña.

Analysis of the publication of articles in the social sciences and humanities based on Current Research Information Systems: the cases of the Universities of Barcelona and Girona

Abstract: Current Research Information Systems from universities can supplement the data offered by international multidisciplinary databases such as WoS or Scopus, especially in disciplines where coverage in these is not sufficiently comprehensive. This study examines characteristics of journal articles in the fields of social sciences and humanities that were published by professors and researchers from two Catalan universities. Data were extracted from databases providing free access to the records of both academic institutions' scholarly production. The results obtained reveal the importance of open access to these kinds of records, as well as the existence of differences among areas of knowledge in quality, level of collaboration, percentage of disclosure, and language of publication, although there is a general trend towards a greater relative use of the English language.

Keywords: Current research information systems; universities; scientific output; Humanities and Social Sciences, Catalonia.

Copyright: (c) 2014 CSIC. Este es un artículo de acceso abierto distribuido bajo los términos de la licencia Creative Commons Attribution-Non Commercial (by-nc) Spain 3.0. 


\section{INTRODUCCIÓN}

Las bases de datos bibliográficas internacionales tales como el Web of Science o Scopus son frecuentemente utilizadas para analizar la producción científica y establecer indicadores bibliométricos (Raan, 2004; Bonaccorsi y otros, 2007). Dichas bases de datos se han mostrado especialmente útiles en disciplinas con un predominio de las publicaciones en lengua inglesa; para el análisis agregado de países, instituciones o áreas de investigación; y para trazar mapas sobre la dinámica de las disciplinas científicas. (Laredo, 2003; Besselaar y otros, 2007).

Sin embargo, diferentes estudios han puesto de relieve las limitaciones en áreas con producción significativa en lenguas diferentes a la inglesa; o bien en disciplinas donde las monografías y otros tipos de documentos tienen un peso considerable (Hicks, 2004; Moed, 2005; Nederhof 2006; Gomez y otros, 2009; Iribarren-Maestro y otros, 2009; Archambault y Larivière, 2010).

Para superar este déficit informativo sobre la producción científica se ha recurrido a bases de datos disciplinares como Medline; currículos; buscadores especializados como Google Scholar; o bien sistemas de archivo abierto y OPACs. (Lepori y otros, 2008; Cañibano y Bozeman, 2009; Torres Salinas y Moed, 2009; Gantman, 2011). Una de las opciones más recientes ha sido el uso de las bases de datos de gestión de la actividad científica de las instituciones académicas (Filippo y otros, 2011).

En España, la mayor parte de las universidades disponen de bases de datos internas que incluyen herramientas más o menos desarrolladas que facilitan la gestión y evaluación de la investigación (Filippo y otros, 2011). Entre los sistemas de información sobre investigación o Current Research Information Systems (CRIS) podemos señalar el Sistema de Información Científica de Andalucía (SICA2); Universitas XXI, liderado por la Universidad Carlos III de Madrid; Científicacvn, de la Universidad de Navarra; Drac, de la Universitat Politècnica de Catalunya; EIN@, de la Universitat Autònoma de Barcelona; y GREC, el más veterano de todos, desarrollado por la Universitat de Barcelona (en adelante UB).

Por su carácter interno el acceso de los investigadores externos a la institución a dichas bases de datos suele estar limitado. Sin embargo, en el contexto español existen algunas excepciones, en concreto las instituciones que utilizan el referido GREC que dispone de una interfaz que permite la consulta pormenorizada por departamentos, facultades, áreas, investigadores y grupos de investigación.

En estos momentos, GREC es utilizada por siete universidades públicas del ámbito lingüístico catalán, una privada, además de otra institución no universitaria. Dos de las universidades públicas son la de Barcelona y la de Girona. La UB es una de las universidades más antiguas e importantes de España, tanto por volumen de estudiantes como por producción científica, según diversas clasificaciones e indicadores tal como se sintetiza en el MetaRanking EC3 de las Universidades Españolas ${ }^{1}$ o los Indicadores bibliométricos de la actividad científica española (Moya, 2013). En términos demográficos, la UB se encuentra situada en el centro de una populosa área metropolitana con unos 5 millones de habitantes. Por su parte, la Universitat de Girona, (UdG) es una institución joven creada a comienzos de los años 90 dentro de un área urbana mucho más reducida, próxima a los 150.000 habitantes. Mientras que, según los datos del Ministerio competente, la UB tenía más de 50.000 estudiantes en el curso 2007-08, la UdG tenía aproximadamente la quinta parte, siendo una de las diez más pequeñas del territorio español. Desde un punto de vista lingüístico, el uso del catalán está más extendido en Girona y su entorno que en el área de Barcelona debido a los diferentes procesos históricos (Burgueño, 1997; Coneixements, 2010).

Tanto en la UdG como en la UB los datos en el GREC son introducidos por el propio personal docente e investigador (PDI), mientras que la supervisión recae en las Oficinas de Transferencia de los Resultados de la Investigación (OTRI). En el caso de la UB, aunque GREC contenía información sobre la producción científica desde mediados de los 90, fue en 1999 cuando se implantó la aplicación de gestión del currículum vitae por internet que permite a los investigadores la actualización de sus datos sobre publicaciones. En el caso de la UdG, hubo una incorporación masiva de datos en el 2005 y la gestión autónoma por parte de los investigadores está disponible desde el 2006.

En los últimos años se está estimulando la introducción de datos por parte del PDI y la mejora de la calidad de los registros. Así la UB está vinculando parcialmente el volumen de carga lectiva del profesorado a la producción científica. Y para su valoración se utilizan los datos correspondientes a los últimos ejercicios anuales según constan en el GREC. Puesto que, naturalmente, no todos los títulos de revista tienen idéntica ponderación, la introducción correcta del título -o del ISSN- es un aspecto que el investigador debe cuidar con mayor atención, así como el año de publicación, puesto que los errores en los datos introducidos pueden generar errores en la valoración de la producción del interesado. Además, también se están desarrollando políticas de vinculación de dicha producción con los repositorios institucionales para la publicación en acceso abierto de los artículos cuando legalmente sea posible.

En el presente artículo se analizan ciertas características de la producción en revistas de ciencias sociales y humanidades -incluidas las artes- en dos universidades catalanas a partir de los datos disponibles en sus CRIS respectivos, con el objetivo de facilitar información complementaria a la 
que puedan aportar las bases de datos internacionales así como otros recursos tales como Google Scholar. Se pretende tanto ofrecer algunos datos que pueden ser representativos de las disciplinas analizadas, como mostrar la utilidad práctica de disponer de tal información para la investigación bibliométrica fuera de las ciencias experimentales y biosanitarias.

Ante la imposibilidad material de analizar los datos de todas las universidades que utilizan el GREC se ha optado por seleccionar la UB atendiendo a su trayectoria histórica, tamaño y peso en la investigación española. El importante volumen de datos obtenido de esta institución se ha comparado con el de la UdG, que, como se ha explicado, es una institución mucho más joven, con un número de alumnos y docentes muy inferior y se halla situada en un territorio con un predominio porcentual del uso del catalán superior al del área de Barcelona.

Entre los aspectos analizados se encuentran la evolución del volumen global de publicación, la calidad de las revistas donde se publica, la importancia relativa de la publicación en la prensa en algunas áreas de conocimiento, así como la lengua de redacción. Al tratarse de un territorio bilingüe, la distribución lingüística de las publicaciones es susceptible de presentar una dinámica propia diferente de la confrontación español versus inglés.

\section{METODOLOGÍA}

En el presente estudio se han obtenido los datos de la producción de artículos de revista de la UB y la UdG de sus respectivas bases de datos GREC. Esta información está disponible para consulta pública$^{2}$. Los datos fueron extraídos durante el mes de octubre de 2012 y exportados a un gestor de hojas de cálculo y posteriormente a una base de datos relacional. Solamente se han contemplado los artículos publicados hasta el año 2011, independientemente del momento en que pudieran haber sido incorporados a la base de datos. De esta manera ha resultado el más antiguo en la UB un documento de 1944 y en la UdG uno de 1971.

Posteriormente se ha procedido a la revisión y depuración de los registros. Puesto que la recuperación de registros se realizó por unidades departamentales, al realizar el análisis por áreas de conocimiento que implican diversos departamentos, como es el caso, por ejemplo, de Derecho o Economía, se eliminaron aquellos que aparecían duplicados. En cambio se han contabilizado como registros independientes cuando se atribuyen a áreas distintas.

Durante el proceso de revisión de los datos se han introducido modificaciones significativas. Por ejemplo, ha sido necesario uniformizar los títulos de las revistas, cuya disparidad se debe no solamente a los cambios en la propia publicación, o a la presencia de títulos alternativos, paralelos, o abreviados, sino también a los errores ortotipográficos producidos durante la introducción original en GREC.
También se ha detectado la presencia de dos o más registros de un mismo documento, generalmente constando un año determinado en uno y el siguiente en el otro. Estos fenómenos se producen incluso tratándose de un solo autor, casos en los que no se puede atribuir a una mera falta de coordinación entre los firmantes del artículo. Después del proceso de revisión se han excluido finalmente 147 registros de artículos que no han podido ser identificados.

Las contribuciones que se introducen en GREC pueden ser de tres tipos: artículos, participaciones en congresos, y otras publicaciones, donde los autores incluyen libros, monografías y otras tipologías documentales. Por tanto no se distingue entre revistas y prensa escrita, lo que solamente puede hacerse a posteriori, controlando el título de la fuente donde se ha publicado.

GREC no dispone de una herramienta para hacer constar la publicación de versiones simultáneas bilingües o de más lenguas de un mismo artículo. Dada la escasa incidencia observada "ad hoc", se ha optado por contabilizar esencialmente únicamente la versión escogida por los autores al introducir los datos en GREC.

Para analizar la calidad de las revistas de cada área, más que recurrir al porcentaje de artículos publicados en revistas del Journal Citation Reports que cubre un escaso $15 \%$ del conjunto de las ciencias sociales (Filippo y otros, 2011), se ha optado por las clasificaciones "ad hoc" CIRC (TorresSalinas y otros, 2010) y CARHUS (Rovira, 2006), estudiando el porcentaje de revistas en cada una de sus categorías. Ambas deberían permitir "a priori" una mayor precisión al abarcar un conjunto mucho más elevado de revistas. Puesto que estas clasificaciones son relativamente recientes, se han contemplado solamente los artículos publicados a partir del año 2001 para evitar un impacto significativo de las revistas no clasificadas debido a su anterior desaparición.

Con la finalidad de obtener un valor sintético sobre la clasificación según la calidad de las revistas en una determinada área se ha calculado el promedio ponderado. Para ello, a los artículos pertenecientes a revistas de las 5 diferentes categorías CIRC (E, A, B, C, D) se les ha asignado un valor natural que parte de 5 para el grupo $\mathrm{E}$ de mayor calidad y termina en 1 para el grupo D. Así, cuanto mayor es la proporción de artículos en revistas de categorías mejor clasificadas, más alta es la media, de manera que un área con valor 5 tendría todas sus revistas clasificadas en la categoría de excelencia (E), mientras que una con valor 1 las tendría todas en el grupo de menor valor (D).

\section{RESULTADOS Y DISCUSIÓN}

Una vez superada la etapa de filtraje, el número total de registros obtenidos entre ambas universidades es de 53.297 , de los cuales un 3,4\% co- 
rresponden a artículos de prensa. Del total, 47.371 $(88,9 \%)$ corresponden a la UB y el resto, 5.926 $(11,1 \%)$ a la UdG.

La distribución cronológica de los documentos en la UB muestra un crecimiento aproximadamente exponencial $\left(R^{2}=0,947\right)$. Sin embargo, hay que considerar no solamente que la introducción de los datos más antiguos en GREC se ha realizado décadas después de la publicación de los trabajos, sino el hecho de que el estímulo para que el profesorado complete la información sobre su producción es relativamente reciente y afecta esencialmente a los últimos años. Por lo tanto, cabe esperar una relativa menor presencia de material antiguo ora por no haberlo introducido personal que actualmente está en activo en dicha universidad, al considerar que no posee demasiado interés práctico y profesional, ora por el hecho de la ausencia de la producción del profesorado que ya haya dejado de tener relación contractual con la UB. En el caso de la UdG, de fundación mucho más reciente, su crecimiento relativo es algo menor y el ajuste a una curva exponencial bastante peor $\left(R^{2}=0,863\right)$. En la figura 1 se recoge la evolución cronológica del número de referencias de artículos en el GREC en ambas universidades. En cualquier caso, se comprueba que el incremento es considerable.

Al fin de estimar la importancia de los artículos frente a otras tipologías documentales se ofrece la tabla I que incluye las cifras agregadas de ambas universidades. En conjunto, los artículos de revistas y prensa constituyen el $29 \%$ de la producción, más bajos que los que se obtienen en las ciencias experimentales. (Filippo y otros, 2011). Destaca también la importancia de los congresos y el predominio en muchas áreas de las publicaciones en libro.

Figura 1. Evolución cronológica del número de artículos

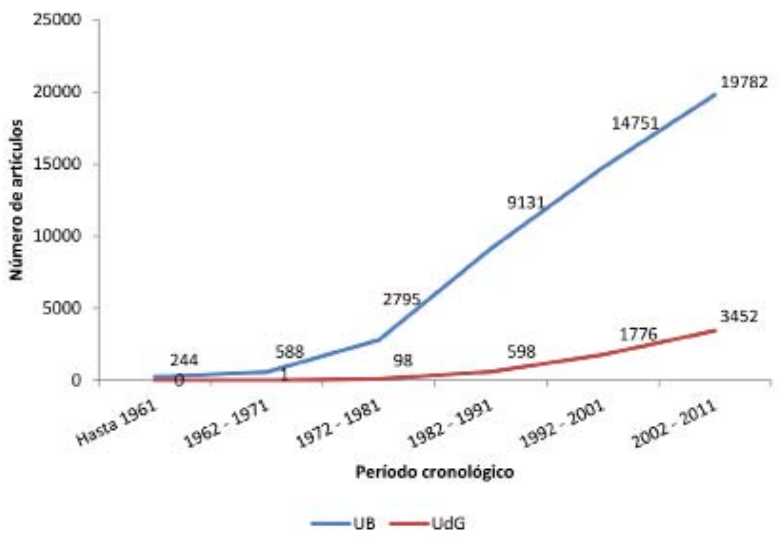

Tabla I. Distribución porcentual por áreas del tipo de documentos producidos en conjunto en la UB y la UdG

\begin{tabular}{lccc}
\hline Área & Artículos en revistas y prensa & Congresos & Libros y otras publicaciones \\
\hline Antropología & $29 \%$ & $34 \%$ & $37 \%$ \\
\hline Bellas artes & $26 \%$ & $18 \%$ & $56 \%$ \\
\hline Comunicación & $26 \%$ & $39 \%$ & $36 \%$ \\
\hline Derecho & $34 \%$ & $26 \%$ & $40 \%$ \\
\hline Documentación & $36 \%$ & $40 \%$ & $24 \%$ \\
\hline Economía & $29 \%$ & $35 \%$ & $35 \%$ \\
\hline Educación & $22 \%$ & $40 \%$ & $38 \%$ \\
\hline Filología & $29 \%$ & $33 \%$ & $38 \%$ \\
\hline Filosofía & $33 \%$ & $35 \%$ & $32 \%$ \\
\hline Geografía & $28 \%$ & $33 \%$ & $39 \%$ \\
\hline Historia del arte & $26 \%$ & $20 \%$ & $55 \%$ \\
\hline Historia y Arqueología & $34 \%$ & $21 \%$ & $45 \%$ \\
\hline Lingüística & $29 \%$ & $37 \%$ & $34 \%$ \\
\hline Psicología & $24 \%$ & $52 \%$ & $24 \%$ \\
\hline Sociología & $29 \%$ & $41 \%$ & $29 \%$ \\
\hline Trabajo social y servicios sociales & $32 \%$ & $46 \%$ & $22 \%$ \\
\hline Total & $29 \%$ & $34 \%$ & $38 \%$ \\
\hline
\end{tabular}


En el caso de la UB, el número de artículos en revistas con un solo autor es de $75,5 \%$, pero este porcentaje ha variado mucho desde el $93,9 \%$ en los años 50, hasta el 68,3\% en el período 2002-2011. Debido a que el volumen global de los artículos de prensa es bajo -ver con detalles más abajo- su influencia es escasa (tabla II). Una situación parecida se ha producido en la UdG en el período 2002-2011, donde algo más de una tercera parte de los artículos han sido firmados por dos o más autores.

Si el cálculo se realiza por áreas de conocimiento, se observan grandes diferencias (tabla III). Así, en Psicología los artículos con un solo autor son menos de la mitad (una tercera parte en la UdG debido a su mayor juventud). Por su parte, Economía presenta en la UdG un porcentaje inferior al 30\%, pero en la UB el valor alcanza el $56,8 \%$. Si se analiza el total de los registros para el período 2002-2011 se observa que tanto en Economía como Geografía hay más artículos publicados en colaboración que con un solo autor, destacando por encima de ellas Psicología donde solamente una cuarta parte de los artículos se han escrito sin colaboración. Puede observarse también cómo Lingüística presenta mayores niveles de colaboración en general que otras disciplinas como Educación o Documentación y se encuentra bien alejado de la Filología, lo que la sitúa en este sentido más en las Ciencias Sociales que en las Humanidades, tal y como ya han mostrado estudios anteriores (Zwaan y Nederhof, 1990; Nederhof y Noyons, 1992; Georgas y Cullars, 2006). En cambio, áreas como Filosofía o Historia del Arte presentan porcentajes por encima del $90 \%$ y en ellas todavía impera de manera abrumadora la costumbre de publicar en solitario.

Si se consideran también los artículos de prensa, lo que no se recoge en la tabla, se acentúa ligeramente el porcentaje de trabajos firmados por un solo autor en aquellas áreas (tabla VI) donde la presencia de este tipo de colaboraciones es más habitual

Sin embargo, puesto que GREC no aporta información que identifique la institución a la que pertenece cada uno de los autores, no es posible establecer un análisis de colaboración.

Tabla II. Distribución cronológica del porcentaje de artículos con un solo autor y media de autores por artículo en la UB y la UdG.

\begin{tabular}{lcccccccc}
\hline & \multicolumn{4}{c}{ UB } & \multicolumn{4}{c}{ UdG } \\
\cline { 2 - 10 } & $\begin{array}{c}\text { \% de artículos con un } \\
\text { solo autor }\end{array}$ & $\begin{array}{c}\text { Media de autores por } \\
\text { artículo }\end{array}$ & $\begin{array}{c}\text { \% de artículos con un } \\
\text { solo autor }\end{array}$ & $\begin{array}{c}\text { Media de autores por } \\
\text { artículo }\end{array}$ \\
\hline Período & revistas & $\begin{array}{c}\text { revistas } \\
+ \\
\text { prensa }\end{array}$ & revistas & $\begin{array}{c}\text { revistas } \\
+ \\
\text { prensa }\end{array}$ & revistas & $\begin{array}{c}\text { revistas } \\
+ \text { prensa }\end{array}$ & revistas & $\begin{array}{c}\text { revistas } \\
+ \text { prensa }\end{array}$ \\
\hline Hasta 1961 & $93,9 \%$ & $93,9 \%$ & 1,1 & 1,1 & - & - & - & - \\
\hline $1962-1971$ & $93,2 \%$ & $93,2 \%$ & 1,1 & 1,1 & - & - & - & - \\
\hline $1972-1981$ & $84,6 \%$ & $84,6 \%$ & 1,2 & 1,2 & $90,1 \%$ & $90,8 \%$ & 1,2 & 1,1 \\
\hline $1982-1991$ & $82,0 \%$ & $82,5 \%$ & 1,3 & 1,3 & $87,0 \%$ & $89,1 \%$ & 1,3 & 1,3 \\
\hline $1992-2001$ & $79,2 \%$ & $79,5 \%$ & 1,4 & 1,4 & $78,7 \%$ & $79,6 \%$ & 1,5 & 1,5 \\
\hline $2002-2011$ & $67,5 \%$ & $68,3 \%$ & 1,7 & 1,7 & $61,4 \%$ & $65,0 \%$ & 1,9 & 1,9 \\
\hline Promedio & $75,5 \%$ & $75,9 \%$ & 1,5 & 1,5 & $69,6 \%$ & $72,2 \%$ & 1,7 & 1,7 \\
\hline
\end{tabular}

Tabla III. Distribución por áreas del porcentaje de artículos en revistas (excluida prensa) con un solo autor y media de autores por artículo en la UB y en la UdG. Período 2002-2011.

\begin{tabular}{|c|c|c|c|c|c|}
\hline \multirow[b]{2}{*}{ Área } & \multicolumn{2}{|c|}{ UB } & \multicolumn{2}{|c|}{ UdG } & \multirow{2}{*}{$\begin{array}{c}\text { Total período 2002-2011 } \\
\begin{array}{c}\% \text { de artículos con un solo } \\
\text { autor }\end{array}\end{array}$} \\
\hline & $\begin{array}{l}\text { \% de artículos } \\
\text { con un solo } \\
\text { autor }\end{array}$ & $\begin{array}{l}\text { Media de } \\
\text { autores por } \\
\text { artículo }\end{array}$ & $\begin{array}{l}\text { \% de artículos } \\
\text { con un solo } \\
\text { autor }\end{array}$ & $\begin{array}{l}\text { Media de } \\
\text { autores por } \\
\text { artículo }\end{array}$ & \\
\hline Antropología & $88,8 \%$ & 1,2 & $80,0 \%$ & 1,2 & $81,9 \%$ \\
\hline Bellas artes & $79,1 \%$ & 1,4 & - & - & $79,1 \%$ \\
\hline Comunicación & - & - & $92,3 \%$ & 1,2 & $90,6 \%$ \\
\hline Derecho & $89,6 \%$ & 1,2 & $87,0 \%$ & 1,2 & $87,4 \%$ \\
\hline Documentación & $64,0 \%$ & 1,6 & - & - & $61,6 \%$ \\
\hline Economía & $56,8 \%$ & 1,7 & $27,0 \%$ & 2,9 & $40,9 \%$ \\
\hline Educación & $72,0 \%$ & 1,5 & $61,6 \%$ & 1,8 & $64,0 \%$ \\
\hline Filología & $90,1 \%$ & 1,2 & $91,4 \%$ & 1,1 & $87,7 \%$ \\
\hline Filosofía & $92,5 \%$ & 1,1 & $99,0 \%$ & 1,0 & $90,6 \%$ \\
\hline Geografía & $57,5 \%$ & 1,7 & $61,8 \%$ & 1,6 & $44,7 \%$ \\
\hline Historia del Arte & $91,7 \%$ & 1,2 & $93,2 \%$ & 1,1 & $91,4 \%$ \\
\hline Historia y Arqueología & $74,8 \%$ & 1,6 & $80,4 \%$ & 1,5 & $69,8 \%$ \\
\hline Lingüística & $63,7 \%$ & 1,9 & $69,0 \%$ & 1,5 & $56,5 \%$ \\
\hline Psicología & $42,0 \%$ & 2,3 & $31,5 \%$ & 3,2 & $24,9 \%$ \\
\hline Sociología & $74,4 \%$ & 1,5 & - & - & $70,4 \%$ \\
\hline Trabajo social y servicios sociales & $57,3 \%$ & 2,4 & - & - & $52,5 \%$ \\
\hline
\end{tabular}


Una de las características importantes susceptible de ser analizada a partir de una base de datos institucional, es el uso de idiomas diferentes al inglés en la publicación de artículos. En términos globales, en cuanto a las Ciencias Sociales y a las Humanidades, en la UB más de la mitad de los artículos se han publicado en español, mientras que aproximadamente una cuarta parte ha sido en catalán. Entre estos dos idiomas acumulan el 84,3\% de los artículos, frente al 11,3\% que se han publicados en inglés. En los datos que se muestran en la tabla IV puede apreciarse que los artículos en francés no llegan al $2 \%$. En otras lenguas presentan porcentajes inferiores. Se aprecia también el número de artículos en las otras lenguas oficiales del Estado español. En la UdG, por su parte, el español y el catalán se han utilizado prácticamente igual. En conjunto suman un $83,1 \%$, valor muy próximo al de la UB. Los artículos en inglés constituyen aproximadamente un 13,4 \% del total, mientras que el resto de idiomas superan ligeramente el $3 \%$. Estos resultados son bastante similares a los que obtenía Gantman (2011) en Ciencias Sociales en Argentina para el periodo 2004-2008, donde un $11 \%$ de los artículos publicados lo fueron en inglés y un $88 \%$ en español.

El estudio por períodos cronológicos de la lengua de publicación de los artículos muestra diferencias importantes entre unos períodos y otros. En el caso de la UB, que recoge algunos registros anteriores a los años 60, se muestra que hasta 1961 casi el $82 \%$ de los artículos estaban escritos en español; los escritos en francés ocupaban el segundo lugar, a una inmensa distancia (9\%); mientras que el catalán y el inglés significaban el $5,7 \%$ y 3,7\% respectivamente.

Durante el período 1962-1971 se produce una leve disminución del porcentaje de artículos en español y un incremento porcentual un poco más importante de los publicados en catalán, situación que se acentúa en las dos siguientes décadas. Puesto que la suma de porcentajes de artículos escritos en inglés y en otras lenguas (esencialmente francés y un poco más tarde italiano y alemán) solamente sufre algunas oscilaciones, lo que muestran los datos es un trasvase parcial del número de publicaciones en español, al catalán, como resultado de la evolución política española y la aparición de vehículos de comunicación bilingües y en catalán. Durante el período 1992-2001 el español sigue su suave declinar, situación que ahora también afecta al catalán con algo más de intensidad, después de varias décadas de crecimiento. Por su parte el inglés alcanza casi un $8 \%$ del total. Este comportamiento es la antesala del último período analizado, el 2002-2011, caracterizado por esa leve, pero continua tendencia del español a perder influencia (56\%); un descenso más acusado del catalán hasta el $21 \%$ y un incremento considerable del inglés $(18 \%)$.

Podemos comparar estos datos con los ofrecidos en Indicadores bibliométricos de la actividad científica española 2010, basado en datos de Scopus. Según estos datos (Moya, 2013), para el período 2006-2010 el 56 \% de la producción en Ciencias

Tabla IV. Distribución según la lengua de los artículos publicados en la UB y la UdG

\begin{tabular}{|c|c|c|c|c|c|c|}
\hline \multirow[b]{2}{*}{ Idioma } & \multicolumn{2}{|c|}{ UB } & \multicolumn{2}{|c|}{ UdG } & \multicolumn{2}{|c|}{ Total } \\
\hline & $\begin{array}{l}\text { Número de } \\
\text { artículos }\end{array}$ & $\begin{array}{c}\% \text { de } \\
\text { artículos }\end{array}$ & $\begin{array}{l}\text { Número de } \\
\text { artículos }\end{array}$ & $\begin{array}{c}\% \text { de } \\
\text { artículos }\end{array}$ & $\begin{array}{l}\text { Número de } \\
\text { artículos }\end{array}$ & $\begin{array}{c}\% \text { de } \\
\text { artículos }\end{array}$ \\
\hline Español & 26982 & $58,6 \%$ & 2217 & $41,2 \%$ & 29199 & $56,9 \%$ \\
\hline Catalán & 11818 & $25,7 \%$ & 2254 & $41,9 \%$ & 14072 & $27,4 \%$ \\
\hline Inglés & 5190 & $11,3 \%$ & 721 & $13,4 \%$ & 5911 & $11,5 \%$ \\
\hline Francés & 891 & $1,9 \%$ & 70 & $1,3 \%$ & 961 & $1,9 \%$ \\
\hline Italiano & 447 & $1,0 \%$ & 58 & $1,1 \%$ & 505 & $1,0 \%$ \\
\hline Alemán & 259 & $0,6 \%$ & 15 & $0,3 \%$ & 274 & $0,5 \%$ \\
\hline Gallego & 224 & $0,5 \%$ & 9 & $0,2 \%$ & 233 & $0,5 \%$ \\
\hline Portugués & 122 & $0,3 \%$ & 13 & $0,2 \%$ & 135 & $0,3 \%$ \\
\hline
\end{tabular}


Sociales es en inglés, un $41 \%$ en español y un $0 \%$ en catalán. En Humanidades un $36 \%$ es en inglés y un $57 \%$ en español. Naturalmente, existe mucha diferencia entre lo que indexa Scopus y el conjunto de toda la producción española.

En el caso de la UdG, hasta el periodo 1992-2001 no coincide realmente con el funcionamiento de la universidad $\mathrm{y}$, por lo tanto es el que debe realmente considerarse. El predominio de los artículos en catalán en los periodos anteriores se debe a la mayor presencia, especialmente en los más antiguos, de publicaciones sobre literatura catalana e historia, con una gran componente regional y mayor tendencia al uso de la lengua vernácula, como se analiza más adelante. Sin embargo, en el período real de funcionamiento de la universidad se aprecia una disminución de los artículos en catalán y un aumento de la producción en inglés.

El análisis del idioma de publicación por áreas arroja diferencias notables. Existen algunas disciplinas donde predomina la publicación en catalán sobre el español, como es el caso de Comunicación, Documentación, Historia y Arqueología. Sin embargo, en la mayoría de casos, es el español la lengua más importante por su volumen de publicación, seguido del catalán. Hay dos importantes excepciones a esta regla: Economía y Psicología. En estas dos áreas de conocimiento, si bien el español es la lengua predominante, en segundo lugar se encuentra el inglés, con un porcentaje agregado para ambas universidades aproximado de un $29 \%$ en ambas disciplinas. En el estudio aludido de Gantman (2011) en Argentina, en Economía el porcentaje era del $24 \%$, pero el de Psicología bajaba hasta el $10 \%$. En cambio Sociología obtenía un $9 \%$ frente al 10,5\% del presente estudio.

En la tabla $V$ se presentan los porcentajes para cada área. Se han desglosado los valores parciales de las diferentes filologías lo que permite comprobar cómo en los casos de la filología hispánica y la filología catalana el predominio respectivo del español y el catalán son abrumadores, lo que no deja de ser natural. El caso de la Filología inglesa y alemana es el único en el que predominan las publicaciones en inglés.

Figura 2. Evolución cronológica del porcentaje de artículos según la lengua de publicación (UB)

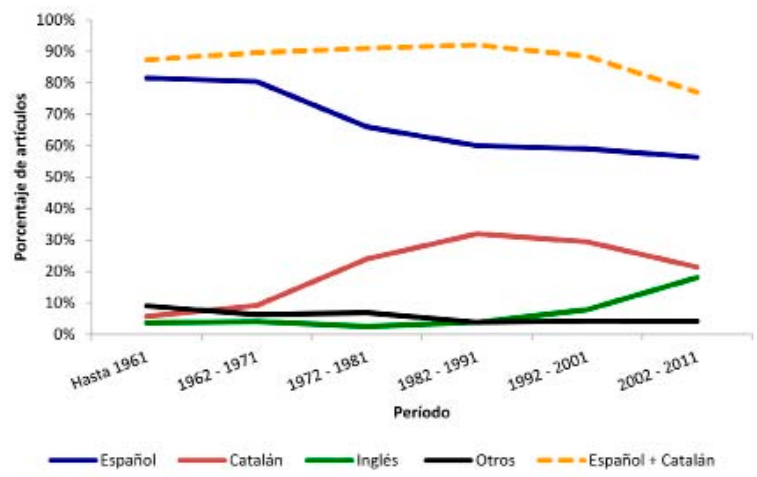

Figura 3. Evolución cronológica del porcentaje de artículos según la lengua de publicación (UdG)

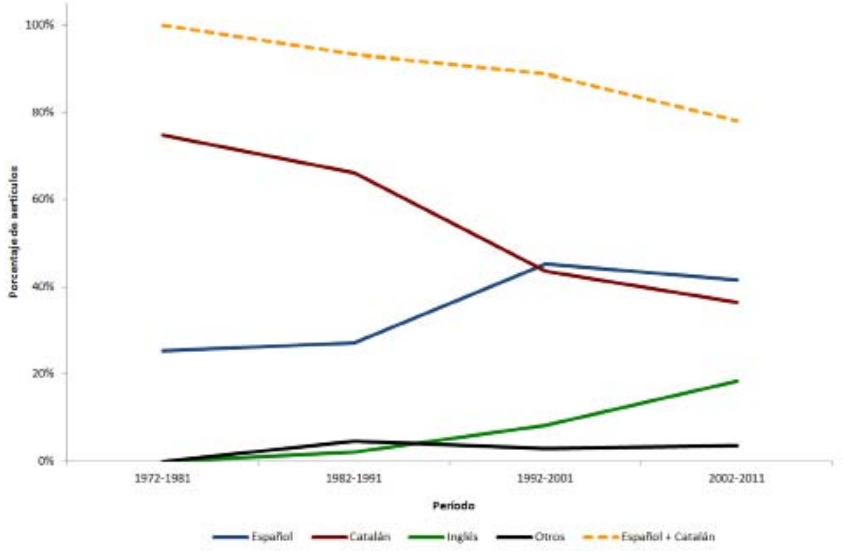


Tabla V. Distribución por áreas de conocimiento del porcentaje de artículos según el idioma de publicación

\begin{tabular}{|c|c|c|c|c|c|c|c|c|c|c|c|c|}
\hline \multirow[b]{2}{*}{$\begin{array}{l}\text { Área de } \\
\text { conocimiento }\end{array}$} & \multicolumn{4}{|c|}{ UB } & \multicolumn{4}{|c|}{ UdG } & \multicolumn{4}{|c|}{ Total } \\
\hline & $\begin{array}{c}\% \\
\text { español }\end{array}$ & $\begin{array}{c}\% \\
\text { catalán }\end{array}$ & $\begin{array}{c}\% \\
\text { inglés }\end{array}$ & $\%$ otros & $\begin{array}{c}\text { \% } \\
\text { español }\end{array}$ & $\begin{array}{c}\% \\
\text { catalán }\end{array}$ & $\begin{array}{c}\% \\
\text { inglés }\end{array}$ & $\begin{array}{c}\% \\
\text { otros }\end{array}$ & $\begin{array}{c}\text { \% } \\
\text { español }\end{array}$ & $\begin{array}{c}\text { \% } \\
\text { catalán }\end{array}$ & $\begin{array}{c}\% \\
\text { inglés }\end{array}$ & $\begin{array}{c}\% \\
\text { otros }\end{array}$ \\
\hline Antropología & $61,3 \%$ & $28,1 \%$ & $5,2 \%$ & $5,3 \%$ & $80,0 \%$ & $20,0 \%$ & $0,0 \%$ & $0,0 \%$ & $61,4 \%$ & $28,1 \%$ & $5,2 \%$ & $5,3 \%$ \\
\hline Bellas artes & $68,3 \%$ & $20,2 \%$ & $5,4 \%$ & $6,1 \%$ & - & - & - & - & $68,3 \%$ & $20,2 \%$ & $5,4 \%$ & $6,1 \%$ \\
\hline Comunicación & - & - & - & - & $26,5 \%$ & $68,4 \%$ & $3,9 \%$ & $1,3 \%$ & $26,5 \%$ & $68,4 \%$ & $3,9 \%$ & $1,3 \%$ \\
\hline Derecho & $82,5 \%$ & $11,4 \%$ & $2,7 \%$ & $3,4 \%$ & $78,5 \%$ & $11,8 \%$ & $2,5 \%$ & $7,2 \%$ & $82,1 \%$ & $11,4 \%$ & $2,6 \%$ & $3,8 \%$ \\
\hline Documentación & $39,6 \%$ & $51,3 \%$ & $8,1 \%$ & $1,0 \%$ & - & - & - & - & $39,6 \%$ & $51,3 \%$ & $8,1 \%$ & $1,0 \%$ \\
\hline Economía & $58,0 \%$ & $14,9 \%$ & $25,5 \%$ & $1,6 \%$ & $32,6 \%$ & $32,1 \%$ & $9,1 \%$ & $57,5 \%$ & $54,4 \%$ & $14,1 \%$ & $29,9 \%$ & $1,6 \%$ \\
\hline Educación & $57,6 \%$ & $35,8 \%$ & $4,3 \%$ & $2,3 \%$ & $49,6 \%$ & $50,3 \%$ & $42,0 \%$ & $5,9 \%$ & $56,6 \%$ & $36,6 \%$ & $4,5 \%$ & $2,3 \%$ \\
\hline $\begin{array}{l}\text { Filología } \\
\text { catalana }\end{array}$ & $10,2 \%$ & $81,0 \%$ & $4,6 \%$ & $4,2 \%$ & $7,9 \%$ & $6,7 \%$ & $87,9 \%$ & $3,6 \%$ & $48,6 \%$ & $34,8 \%$ & $14,4 \%$ & $2,2 \%$ \\
\hline Filología clásica & $54,7 \%$ & $33,5 \%$ & $3,8 \%$ & $8,0 \%$ & $1,3 \%$ & $1,9 \%$ & $97,1 \%$ & $0,0 \%$ & $50,9 \%$ & $38,1 \%$ & $3,5 \%$ & $7,5 \%$ \\
\hline $\begin{array}{l}\text { Filología } \\
\text { hispánica }\end{array}$ & $95,0 \%$ & $3,4 \%$ & $1,0 \%$ & $0,6 \%$ & $80,6 \%$ & $81,0 \%$ & $13,2 \%$ & $4,6 \%$ & $93,7 \%$ & $4,4 \%$ & $1,3 \%$ & $0,6 \%$ \\
\hline $\begin{array}{l}\text { Filología } \\
\text { inglesa y } \\
\text { alemana }\end{array}$ & $30,7 \%$ & $9,8 \%$ & $53,3 \%$ & $6,2 \%$ & - & - & - & - & $30,7 \%$ & $9,8 \%$ & $53,3 \%$ & $6,2 \%$ \\
\hline $\begin{array}{l}\text { Filología } \\
\text { románica }\end{array}$ & $51,7 \%$ & $19,8 \%$ & $0,9 \%$ & $27,7 \%$ & $6,3 \%$ & $46,3 \%$ & $20,0 \%$ & $27,5 \%$ & $49,6 \%$ & $21,0 \%$ & $1,8 \%$ & $27,7 \%$ \\
\hline $\begin{array}{l}\text { Filología } \\
\text { semítica }\end{array}$ & $61,6 \%$ & $11,8 \%$ & $14,2 \%$ & $12,4 \%$ & $12,2 \%$ & $87,8 \%$ & $0,0 \%$ & $0,0 \%$ & $60,2 \%$ & $13,8 \%$ & $13,9 \%$ & $12,1 \%$ \\
\hline Filología (total) & $51,7 \%$ & $28,8 \%$ & $9,4 \%$ & $10,2 \%$ & $22,7 \%$ & $68,4 \%$ & $4,8 \%$ & $4,1 \%$ & $49,3 \%$ & $32,1 \%$ & $9,0 \%$ & $9,6 \%$ \\
\hline Filosofía & $62,2 \%$ & $18,8 \%$ & $15,5 \%$ & $3,4 \%$ & $39,0 \%$ & $45,1 \%$ & $11,8 \%$ & $4,1 \%$ & $60,5 \%$ & $20,7 \%$ & $15,3 \%$ & $3,5 \%$ \\
\hline Geografía & $55,7 \%$ & $30,7 \%$ & $10,4 \%$ & $3,2 \%$ & $38,2 \%$ & $44,1 \%$ & $8,8 \%$ & $8,8 \%$ & $55,4 \%$ & $31,0 \%$ & $10,4 \%$ & $3,3 \%$ \\
\hline $\begin{array}{l}\text { Historia del } \\
\text { arte }\end{array}$ & $45,7 \%$ & $48,0 \%$ & $2,4 \%$ & $3,8 \%$ & $49,7 \%$ & $41,2 \%$ & $2,0 \%$ & $7,1 \%$ & $46,5 \%$ & $46,7 \%$ & $2,3 \%$ & $4,5 \%$ \\
\hline $\begin{array}{l}\text { Historia y } \\
\text { Arqueología }\end{array}$ & $37,7 \%$ & $50,2 \%$ & $7,5 \%$ & $4,6 \%$ & $20,1 \%$ & $75,4 \%$ & $1,7 \%$ & $2,8 \%$ & $34,6 \%$ & $54,6 \%$ & $6,5 \%$ & $4,3 \%$ \\
\hline Lingüística & $55,1 \%$ & $21,1 \%$ & $17,2 \%$ & $6,6 \%$ & $48,3 \%$ & $25,9 \%$ & $12,1 \%$ & $13,8 \%$ & $54,7 \%$ & $21,4 \%$ & $16,8 \%$ & $7,1 \%$ \\
\hline Psicología & $61,2 \%$ & $6,9 \%$ & $30,2 \%$ & $1,7 \%$ & $58,8 \%$ & $15,0 \%$ & $25,2 \%$ & $1,0 \%$ & $60,9 \%$ & $7,7 \%$ & $29,7 \%$ & $1,7 \%$ \\
\hline Sociología & $72,1 \%$ & $15,3 \%$ & $10,6 \%$ & $2,0 \%$ & - & - & - & - & $72,1 \%$ & $15,3 \%$ & $10,6 \%$ & $2,0 \%$ \\
\hline $\begin{array}{l}\text { Trabajo social } \\
\text { y servicios } \\
\text { sociales }\end{array}$ & $64,5 \%$ & $22,6 \%$ & $9,8 \%$ & $3,0 \%$ & - & - & - & - & $64,5 \%$ & $22,6 \%$ & $9,8 \%$ & $3,0 \%$ \\
\hline
\end{tabular}


En el análisis de los títulos se hace patente la presencia de prensa diaria o semanal. En el caso de la UB este tipo de documentos corresponde al 2,7\% de los artículos, aunque hay notables diferencias entre áreas, tal y como muestra la tabla VI, destacando el 7,3\% de Bellas Artes, el 7,2 \% de Filosofía y el $5,2 \%$ de Historia y Arqueología. En la UdG el porcentaje total es más alto, llegando hasta un 9,2\%. La distribución por áreas muestra aquí mayores desigualdades, ya que en el caso de Historia del Arte el $52,8 \%$ de los artículos han sido publicados en prensa diaria o semanal. En el caso de Filología el porcentaje es del 7,7\%, mientras que en el resto de áreas el porcentaje es más bajo. En ambas universidades se aprecia un descenso de la importancia en porcentaje de este tipo de publicaciones que, sin embargo, han venido desempeñando cierta función de comunicación científica en algunas áreas de Humanidades y Ciencias Sociales más allá de la mera divulgación, como lo atestigua la bibliografía de análisis de citas (Ardanuy y otros, 2009).

La tabla VII muestra los títulos de los periódicos con más de 10 artículos publicados entre las dos universidades. Se observa un predominio destacado de La Vanguardia editado en Barcelona, seguido a cierta distancia por El País de Madrid. En tercer lugar se encuentra el semanario El Temps, editado en Valencia.

Tabla VI. Distribución por áreas de conocimiento del porcentaje de artículos publicados en prensa diaria o semanal.

\begin{tabular}{|c|c|c|c|c|c|}
\hline \multirow[b]{2}{*}{ Área } & \multicolumn{2}{|l|}{ UB } & \multicolumn{2}{|l|}{ UdG } & Total \\
\hline & $\begin{array}{l}\text { Total de artículos } \\
\text { (revistas + prensa) }\end{array}$ & $\begin{array}{l}\text { \% artículos } \\
\text { de prensa }\end{array}$ & $\begin{array}{l}\text { Total de artículos } \\
\text { (revistas + prensa) }\end{array}$ & $\begin{array}{l}\text { \% artículos } \\
\text { de prensa }\end{array}$ & $\begin{array}{l}\text { \% artículos } \\
\text { de prensa }\end{array}$ \\
\hline Antropología & 1514 & $0,9 \%$ & 10 & $0,0 \%$ & $0,9 \%$ \\
\hline Bellas artes & 1329 & $7,3 \%$ & - & - & $7,3 \%$ \\
\hline Comunicación & - & - & 161 & $3,7 \%$ & $3,7 \%$ \\
\hline Derecho & 6491 & $3,6 \%$ & 681 & $0,4 \%$ & $3,3 \%$ \\
\hline Documentación & 807 & $3,2 \%$ & - & . & $3,2 \%$ \\
\hline Economía & 4872 & $3,3 \%$ & 779 & $4,0 \%$ & $3,4 \%$ \\
\hline Educación & 5646 & $1,3 \%$ & 896 & $2,2 \%$ & $1,4 \%$ \\
\hline Filología & 8761 & $1,1 \%$ & 854 & $7,7 \%$ & $1,7 \%$ \\
\hline Filosofía & 2656 & $7,2 \%$ & 196 & $0,5 \%$ & $6,7 \%$ \\
\hline Geografía & 1759 & $0,6 \%$ & 35 & $2,9 \%$ & $0,6 \%$ \\
\hline Historia del arte & 1529 & $3,0 \%$ & 745 & $52,8 \%$ & $19,3 \%$ \\
\hline Historia y Arqueología & 4992 & $5,2 \%$ & 1031 & $2,6 \%$ & $4,8 \%$ \\
\hline Lingüística & 805 & $1,7 \%$ & 58 & $0,0 \%$ & $1,6 \%$ \\
\hline Psicología & 4228 & $0,4 \%$ & 480 & $0,0 \%$ & $0,4 \%$ \\
\hline Sociología & 1748 & $1,2 \%$ & - & - & $1,2 \%$ \\
\hline Trabajo social y servicios sociales & 234 & $0,0 \%$ & - & - & $0,0 \%$ \\
\hline Total & 47.371 & $2,7 \%$ & 5926 & $9,2 \%$ & $3,4 \%$ \\
\hline
\end{tabular}

Tabla VII. Cabeceras de prensa con más artículos publicados

\begin{tabular}{lcccc}
\hline Título & $\begin{array}{c}\text { Número de } \\
\text { artículos }\end{array}$ & $\begin{array}{c}\text { Localidad principal de } \\
\text { edición }\end{array}$ & Ámbito principal & Periodicidad \\
\hline La Vanguardia & 500 & Barcelona & España & Diario \\
\hline El País & 295 & Madrid & España & Siario \\
\hline El Temps & 190 & Valencia & Dominio lingǘstico catalán & Demario \\
\hline Avui & 154 & Barcelona & Cataluña & Diario \\
\hline El Periódico de Catalunya & 76 & Barcelona & Cataluña & Diario \\
\hline El Punt & 76 & Gerona & Cataluña & Diario \\
\hline Diari de Barcelona & 63 & Barcelona & Barcelona (local) & Diario \\
\hline Expansión & 57 & Madrid & España & Demanario \\
\hline El Món & 53 & Barcelona & Cataluña (regional) & Diario \\
\hline Diari de Girona & 39 & Gerona & Girona (provincial) & Diario \\
\hline ABC & 26 & Madrid & España & Semanario \\
\hline La Voz de Galicia & 18 & La Coruña & Galicia & Diario \\
\hline La Veu de I'Anoia & 17 & Igualada & Anoia (comarcal) & Diario \\
\hline Sport & 15 & Barcelona & España & Semanario \\
\hline Última Hora & 14 & Palma de Mallorca & Baleares & Sitges (local) \\
\hline Eco de Sitges & 12 & Sitges & &
\end{tabular}


Naturalmente la dispersión de revistas donde se han publicado los artículos es enorme. Se necesitan 69 títulos de revistas para recoger el 25\% de artículos publicados, 409 para llegar al 50\%, alcanzando el $100 \%$ con 10.276 títulos diferentes. En la tabla VIII se han recogido los primeros 25 títulos y el número de artículos publicados.

Para estimar la calidad de las revistas se ha recurrido a las clasificaciones CIRC y CARHUS, estudiando la distribución porcentual en cada una de sus categorías de los artículos publicados a partir del año 2001. Una de las primeras cuestiones que se observa es que tanto en la UB como en la UdG algo más del $50 \%$ de los artículos han sido publicados en revistas que no tienen ninguna categoría asignada en CARHUS. Este último sistema de clasificación desarrollado por la Agencia de Gestión de Ayudas Universitarias y de Investigación de Cataluña, cubre en la actualidad 4.953 títulos (Rovira, 2006; AGAUR, 2011). Sin embargo, esta dificultad no la encontramos en CIRC, puesto que al margen de las 20.756 revistas que clasifica explícitamen- te, establece la categoría D para todas aquellas publicaciones no incluidas en las otras categorías y que se consideran de dudoso estatus científico (Torres Salinas y otros, 2010). Mientras que en algunas áreas el porcentaje de artículos de revistas del grupo $D$ supera a los que no se encuentran en CARHUS, en otras la situación es claramente diferente, lo que muestra que las categorías de ambas clasificaciones no son directamente asimilables.

Asignando como se ha expuesto anteriormente un valor entre 1 y 5 según la categoría CIRC ( $E$, A, B, C, D) a la que pertenecen las revistas, la UB obtiene una media CIRC de 2,10, frente al 2,03 de la UdG, una diferencia escasa cercana al 3,5\%.

El análisis por áreas muestra de nuevo sensibles diferencias. En el caso de la UB (tabla IX) la media más alta la obtiene psicología $(2,97)$, con $15,8 \%$ de los trabajos en el grupo de excelencia. En el extremo contrario se encuentran Bellas Artes e Historia del Arte, con medias muy semejantes del 1,57 y 1,58 respectivamente y presencias testimoniales en el grupo de revistas de excelencia.

Tabla VIII. Revistas con más artículos publicados

\begin{tabular}{|c|c|c|c|}
\hline Título & $\begin{array}{l}\text { Número de } \\
\text { artículos }\end{array}$ & $\%$ & $\%$ acumulado \\
\hline L'Avenç. Revista catalana d'història & 676 & $1,3 \%$ & $1,3 \%$ \\
\hline Cuadernos de Pedagogía & 595 & $1,2 \%$ & $2,5 \%$ \\
\hline Revista Jurídica de Catalunya & 463 & $0,9 \%$ & $3,4 \%$ \\
\hline Anuario de Psicología & 435 & $0,8 \%$ & $4,2 \%$ \\
\hline Aula de Innovación Educativa & 431 & $0,8 \%$ & $5,0 \%$ \\
\hline Guix. Elements d'Acció Educativa & 427 & $0,8 \%$ & $5,9 \%$ \\
\hline Temps d'Educació & 398 & $0,8 \%$ & $6,7 \%$ \\
\hline Anuari de Filologia & 392 & $0,8 \%$ & $7,4 \%$ \\
\hline Serra d'Or & 363 & $0,7 \%$ & $8,1 \%$ \\
\hline Revista de Girona & 356 & $0,7 \%$ & $8,8 \%$ \\
\hline Aula Orientalis. Ed. Ausa & 284 & $0,6 \%$ & $9,4 \%$ \\
\hline Tribuna Social & 276 & $0,5 \%$ & $9,9 \%$ \\
\hline Revista de Catalunya & 275 & $0,5 \%$ & $10,4 \%$ \\
\hline El Ciervo. Revista Mensual de Pensamiento y Cultura & 270 & $0,5 \%$ & $11,0 \%$ \\
\hline Perspectiva Escolar & 217 & $0,4 \%$ & $11,4 \%$ \\
\hline Revista española de Derecho internacional & 204 & $0,4 \%$ & $11,8 \%$ \\
\hline Escola Catalana & 191 & $0,4 \%$ & $12,1 \%$ \\
\hline Mientras Tanto & 186 & $0,4 \%$ & $12,5 \%$ \\
\hline Estudis Romànics & 181 & $0,4 \%$ & $12,9 \%$ \\
\hline Els Marges & 178 & $0,3 \%$ & $13,2 \%$ \\
\hline Pyrenae: Revista de Prehistòria i Antiguitat de la Mediterrània Occidental & 178 & $0,3 \%$ & $13,5 \%$ \\
\hline Autonomies. Revista Catalana de Dret Públic & 159 & $0,3 \%$ & $13,9 \%$ \\
\hline El Viejo Topo & 159 & $0,3 \%$ & $14,2 \%$ \\
\hline Acta Historica et Archaeologica Mediaevalia & 157 & $0,3 \%$ & $14,5 \%$ \\
\hline
\end{tabular}


En la UdG (tabla X), dejando de lado Geografía, con una muestra muy pequeña de artículos, sobresale Economía $(2,68)$ con un $13,4 \%$ artículos en el grupo $\mathrm{E}$ y un $30,4 \%$ en el grupo $\mathrm{A}$.

Si consideramos los artículos de ambas universidades conjuntamente, las disciplinas con menos artículos en el grupo A y E son Derecho (2,2 \%), Historia del Arte (3,9\%) y Bellas Artes (4,2\%). Estas dos últimas también presentan un mayor número de artículos en el grupo D, 58,2 \% y 65,9 \% respectivamente. En el extremo opuesto se encuentra Psicología, que ocupa el primer lugar con $14,5 \%$ en el grupo $E$ y un total del $45,8 \%$ sumando $E$ y $A$. Compárense tales valores con la media para todas las disciplinas del 3'9 \% y 16,9\% respectivamente. Economía arroja un resultado de $6,8 \%$ en el grupo $\mathrm{E}$ y $32,6 \%$ en $E$ más $A$. La media en el grupo $D$ es de
43,3\%, encontrándose en el área de Psicología un 28 ' $2 \%$ de los artículos en dicho grupo y un $43,8 \%$ en el caso de Economía. Sin embargo, el procedimiento permite mostrar que existe una diferencia cualitativa entre las dos. Mientras que Psicología presenta la cifra más pequeña de artículos en el grupo D de CIRC, Economía se encuentra unas décimas por encima de la media. Si consideramos que el grupo $D$, según los creadores de la clasificación «estaría conformado por todas aquellas publicaciones [...] con un dudoso status científico» (Torres Salinas y otros, 2010), puede colegirse que las publicaciones del área de Economía tienen una mayor vocación docente y/o divulgativa que las de Psicología. Obsérvese como paralelamente Psicología presenta el porcentaje más bajo de artículos en prensa y, en cambio, el guarismo de Economía es exactamente el correspondiente a la media.

Tabla IX. Distribución del porcentaje de revistas en cada área según las categorías CIRC y CARHUS (UB)

\begin{tabular}{|c|c|c|c|c|c|c|c|c|c|c|c|c|}
\hline \multirow{2}{*}{ Área } & \multirow{2}{*}{$\begin{array}{c}\text { Total } \\
\text { artículos }\end{array}$} & \multicolumn{5}{|c|}{ \% Categoría CIRC } & \multirow{2}{*}{$\begin{array}{l}\text { Media } \\
\text { CIRC }\end{array}$} & \multicolumn{5}{|c|}{ \% Categoría CARHUS } \\
\hline & & E & A & B & C & D & & A & B & C & D & $\begin{array}{l}\text { Sin } \\
\text { clasificar }\end{array}$ \\
\hline Antropología & 528 & 1,5 & 10,0 & 13,3 & 32,6 & 42,6 & 1,95 & 4,4 & 18,8 & 17,4 & 4,0 & 55,5 \\
\hline Bellas artes & 549 & 0,4 & 3,8 & 14,6 & 15,3 & 65,9 & 1,57 & 3,3 & 1,6 & 17,3 & 3,3 & 74,5 \\
\hline Derecho & 3016 & 0,2 & 2,1 & 28,7 & 21,9 & 47,2 & 1,86 & 7,7 & 9,7 & 32,3 & 2,7 & 47,7 \\
\hline Documentación & 504 & 1,0 & 18,7 & 29,6 & 21,0 & 29,8 & 2,40 & 3,4 & 38,5 & 15,7 & 2,0 & 40,5 \\
\hline Economía & 2734 & 5,4 & 24,8 & 14,8 & 10,6 & 44,3 & 2,37 & 7,4 & 9,5 & 11,7 & 3,2 & 68,2 \\
\hline Educación & 2729 & 0,5 & 6,2 & 18,2 & 27,2 & 48,0 & 1,84 & 5,7 & 11,8 & 29,7 & 2,8 & 50,0 \\
\hline Filología & 3214 & 1,4 & 9,2 & 25,0 & 20,6 & 43,8 & 2,04 & 14,2 & 9,6 & 20,2 & 4,0 & 52,0 \\
\hline Filosofía & 1098 & 6,4 & 21,6 & 25,1 & 13,8 & 33,1 & 2,54 & 20,9 & 7,7 & 16,9 & 1,7 & 52,6 \\
\hline Geografía & 639 & 4,7 & 21,6 & 24,9 & 16,4 & 32,4 & 2,50 & 3,0 & 19,6 & 14,4 & 1,1 & 62,0 \\
\hline Historia del arte & 540 & 0,4 & 3,5 & 8,1 & 29,4 & 58,5 & 1,58 & 2,6 & 1,9 & 24,8 & 3,3 & 67,4 \\
\hline Historia y Arqueología & 1733 & 4,8 & 8,9 & 21,6 & 21,0 & 43,6 & 2,10 & 15,7 & 6,9 & 21,8 & 4,0 & 51,6 \\
\hline Lingüística & 444 & 4,5 & 3,2 & 26,4 & 14,0 & 52,0 & 1,94 & 18,2 & 7,0 & 27,0 & 3,4 & 44,4 \\
\hline Psicología & 2096 & 15,8 & 32,2 & 11,0 & 14,8 & 26,2 & 2,97 & 16,7 & 27,8 & 8,3 & 0,3 & 46,9 \\
\hline Sociología & 860 & 2,2 & 11,7 & 19,5 & 24,7 & 41,9 & 2,08 & 5,2 & 14,7 & 18,6 & 12,6 & 49,0 \\
\hline $\begin{array}{l}\text { Trabajo social y } \\
\text { servicios sociales }\end{array}$ & 167 & 3,6 & 7,2 & 5,4 & 26,9 & 56,9 & 1,74 & 4,2 & 6,6 & 44,9 & 0,0 & 44,3 \\
\hline Total UB & 20851 & 3,8 & 13,1 & 20,4 & 19,8 & 43,0 & 2,15 & 10,2 & 12,4 & 20,8 & 3,2 & 53,5 \\
\hline
\end{tabular}

Tabla X. Distribución del porcentaje de revistas en cada área según las categorías CIRC y CARHUS (UdG)

\begin{tabular}{|c|c|c|c|c|c|c|c|c|c|c|c|c|}
\hline \multirow{2}{*}{ Área } & \multirow{2}{*}{$\begin{array}{c}\text { Total } \\
\text { artículos }\end{array}$} & \multicolumn{5}{|c|}{ \% Categoría CIRC } & \multirow{2}{*}{$\begin{array}{l}\text { Media } \\
\text { CIRC }\end{array}$} & \multicolumn{5}{|c|}{$\%$ Categoría CARHUS } \\
\hline & & $\mathbf{E}$ & A & B & C & D & & A & B & C & D & $\begin{array}{c}\text { Sin } \\
\text { clasificar }\end{array}$ \\
\hline Antropología & 8 & 0,0 & 0,0 & 12,5 & 0,0 & 87,5 & 1,25 & 0,0 & 0,0 & 25,0 & 0,0 & 75,0 \\
\hline Comunicación & 89 & 0,0 & 10,1 & 25,8 & 25,8 & 38,2 & 2,08 & 1,1 & 10,1 & 23,6 & 9,0 & 56,2 \\
\hline Derecho & 449 & 0,4 & 1,3 & 29,6 & 16,0 & 52,6 & 1,81 & 10,9 & 7,1 & 29,0 & 2,4 & 50,6 \\
\hline Economía & 575 & 13,4 & 30,3 & 8,2 & 7,0 & 41,2 & 2,68 & 10,8 & 9,4 & 5,7 & 2,8 & 71,3 \\
\hline Educación & 615 & 0,7 & 7,5 & 14,1 & 25,5 & 52,2 & 1,79 & 6,8 & 8,3 & 26,5 & 4,6 & 53,8 \\
\hline Filología & 480 & 0,0 & 8,3 & 13,8 & 36,0 & 41,9 & 1,89 & 13,3 & 3,3 & 26,3 & 16,9 & 40,2 \\
\hline Filosofía & 126 & 5,6 & 13,5 & 15,9 & 15,1 & 50,0 & 2,10 & 12,7 & 10,3 & 15,9 & 5,6 & 55,6 \\
\hline Geografía & 8 & 25,0 & 12,5 & 37,5 & 0,0 & 25,0 & 3,13 & 25,0 & 25,0 & 37,5 & 0,0 & 12,5 \\
\hline Historia del Arte & 177 & 1,7 & 2,3 & 13,0 & 26,0 & 57,1 & 1,66 & 4,0 & 0,6 & 15,8 & 13,6 & 66,1 \\
\hline Historia y Arqueología & 453 & 0,4 & 6,4 & 12,8 & 36,4 & 43,9 & 1,83 & 5,3 & 4,6 & 21,6 & 7,1 & 61,4 \\
\hline Lingüística & 40 & 0,0 & 0,0 & 17,5 & 42,5 & 40,0 & 1,78 & 10,0 & 0,0 & 55,0 & 0,0 & 35,0 \\
\hline Psicología & 326 & 5,5 & 26,4 & 9,5 & 17,2 & 41,4 & 2,37 & 9,2 & 23,3 & 7,1 & 0,3 & 60,1 \\
\hline Total UdG & 3346 & 3,4 & 12,3 & 14,9 & 23,0 & 46,4 & 2,03 & 9,0 & 8,2 & 20,0 & 6,2 & 56,6 \\
\hline
\end{tabular}


La figura 4 presenta en un gráfico bidimensional las posiciones relativas de las diferentes áreas atendiendo a las dos variables aludidas en el párrafo anterior, el porcentaje de revistas en las categorías de mayor calidad (E y A de CIRC) y el porcentaje de artículos publicados en revistas, es decir, no publicados en la prensa diaria o semanal. Naturalmente, cuando las áreas se encuentran muy próximas implica un comportamiento semejante en cuanto a los mecanismos de publicación, por lo que hace a las dos variables analizadas. Es el caso, por ejemplo, de Lingüística y Educación; $o$, en menor medida de Derecho y Bellas Artes. En la figura se distinguen con facilidad las singularidades mencionadas de Psicología y Economía. También sobresale Filosofía, en cuanto al porcentaje de artículos en revistas en las mejores categorías de CIRC. Pero a la vez muestra un porcentaje relativamente elevado de artículos en la prensa escrita. Derecho, tal y como hemos comentado, muestra el guarismo más bajo de artículos en las categorías reinas de CIRC, pero en cambio ocupa una posición de mayor centralidad relativa en cuanto a los artículos en prensa.

\section{CONCLUSIONES}

Los sistemas de información sobre investigación y actividad científica así como otras bases de datos de institucionales están concebidos en su origen para la explotación interna de la propia organización. En el caso específico de la producción científica, su objetivo es facilitar su análisis con diferentes niveles de agregación: investigadores, departamentos, grupos de investigación, áreas de conocimiento, etc. Sin embargo, la calidad de los análisis $y$, por tanto, las conclusiones que puedan desprenderse, dependen de la precisión de los datos introducidos. En este sentido es necesario fortalecer tanto los incentivos para que el profesorado sea escrupuloso en la introducción de datos, como la tarea de validación realizada por las OTRI.
Por otra parte, las universidades públicas, como toda la administración pública, precisan de mecanismos que aseguren la transparencia y la rendición de cuentas ante los miembros de su comunidad y la sociedad en general. Puesto que en gran parte su actividad se financia con recursos públicos, parece razonable exigir que dichos mecanismos permitan conocer con suficiente detalle la producción científica tanto individualmente como de las distintas unidades en las que se organiza el PDI (departamentos, facultades, institutos, ...). En este sentido, como demuestra este trabajo, la posibilidad de la consulta de libre acceso a GREC -o una base de datos equivalente- por parte de investigadores e interesados, facilita la transparencia, tanto externa como interna en la institución, mientras que los meros listados de producción, si bien suministran los datos en sentido estricto, no facilitan las comparaciones.

Si nos centramos en los datos cuantitativos, se observa la importancia de las participaciones en congresos que supera globalmente a las de los artículos y se acerca a la del conjunto de publicaciones en libros. Este elevado porcentaje puede merecer un futuro estudio detallado de tal tipología documental.

Considerando el conjunto de las disciplinas de Ciencias Sociales y Humanidades, tanto la UB y la UdG escriben para una audiencia más bien regional que lee principalmente en español y catalán. No es ajeno a ello el hecho de que la mayor parte de publicaciones en español en Ciencias Sociales y Humanidades hayan sido tradicionalmente publicadas básicamente en español, seguidas a gran distancia por el catalán (Osca Lluch y Mateo Marquina, 2003).

En el caso de la UB, con una larga trayectoria histórica y muy representativa por el volumen de alumnos y la importancia de su producción a nivel español, se observa que, cuando fue posible, se

Figura 4. Distribución bidimensional de las disciplinas según porcentaje de artículos publicados en revistas (no en prensa) y artículos publicados en las categorías E y A de CIRC.

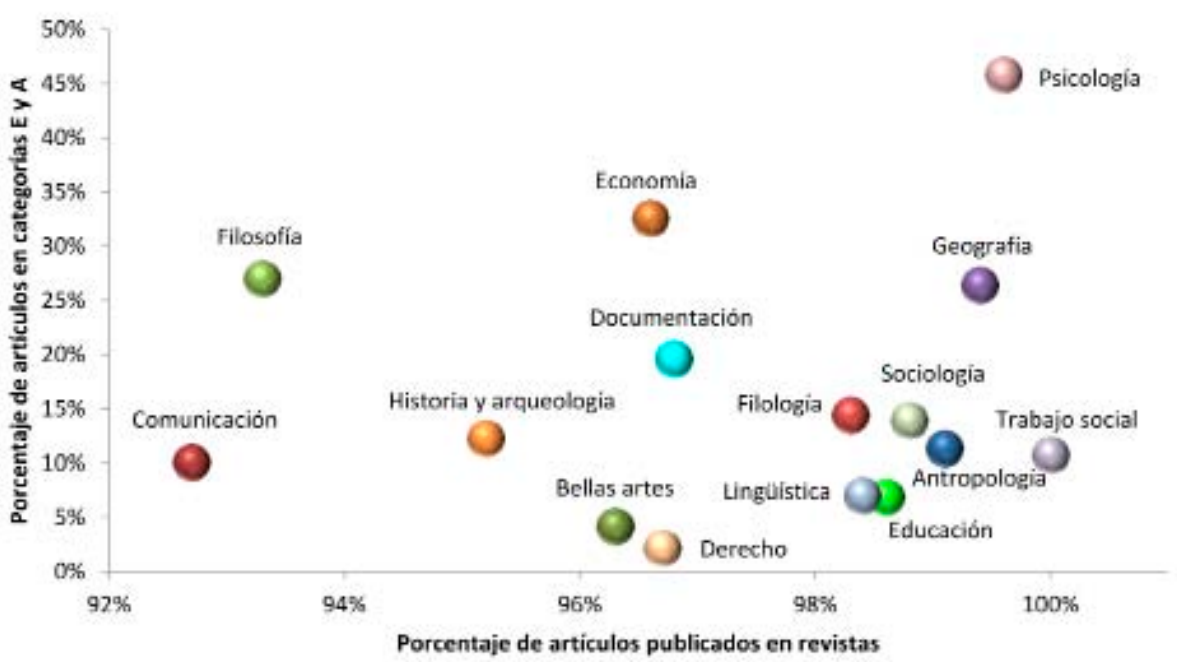


produjo un mero trasvase de artículos publicados en español al catalán. El hecho de que el porcentaje de artículos publicados en conjunto en español y catalán se sitúe en torno al $85 \%$, con un valor muy similar en la UdG, una institución mucho más joven, de volumen reducido y en un entorno demográfico con un peso más elevado del catalán, sugiere que tales datos son extrapolables a otras universidades españolas, donde el conjunto de la producción en español -o de español más otra lengua vernácula, de existir- puede ser muy similar. Obsérvese que en el caso de la producción argentina en Ciencias Sociales dicho guarismo es relativamente similar, del 88\% (Gantman, 2011).

Ahora bien, si se utiliza el uso de la lengua inglesa como criterio significativo de internacionalización de las Ciencias Sociales y las Humanidades, los incrementos porcentuales importantes en los últimos años muestran un esfuerzo por aumentar la visibilidad y el impacto de los estudios publicados, de lo que naturalmente no debería estar exento de responsabilidad el llamado "efecto ANECA" (Soriano, 2008). Esta voluntad se percibe también en la disminución de las publicaciones en catalán, que es más considerable que en español, fenómeno que en ausencia de causas sociopolíticas destacables, puede atribuirse a la existencia de un mayor número de revistas en español que en catalán $y$ al hecho de que las primeras gozan de una mayor visibilidad e impacto.

Con respecto a las diferentes áreas o disciplinas de conocimiento, se confirma una mayor proyección internacional de la Psicología y de la Economía, así como una presencia importante de artículos en revistas del grupo de excelencia en la clasificación CIRC. Por otra parte, la comparación de los porcentajes de artículos en la categoría $\mathrm{D}$ de CIRC, así como en la prensa escrita, facilitan información sobre la tendencia a publicar artículos con finalidad docente o divulgativa. Así, por ejemplo, se observa que Bellas Artes y Derecho muestran un perfil semejante en cuanto a estas dos dimensiones. Precisamente con el objetivo de distinguirlas con mayor claridad hay iniciativas recientes, como la de la UB, de establecer para cada artículo si la finalidad principal es investigación, docencia, divulgación u otras, sin que necesariamente tengan que ser mutuamente excluyentes.

Finalmente, concluyamos que se detectan incrementos muy ligeros del nivel de colaboración en áreas de Humanidades tales como Filosofía, Filología o Historia del Arte. Así que debe afirmarse que se sigue publicando en solitario. Sin embargo, en las áreas de Psicología, Economía y Geografía, dentro de las Ciencias Sociales, la publicación en colaboración es ya mayoritaria.

\section{NOTAS}

[1] http://wdb.ugr.es/ elrobin/. Fecha de comprobación de url: 19 de febrero de 2014

[2] UB: https://webgrec.ub.edu/. UdG: https://webgrec. udg.edu. En ambos casos consulta 14 de febrero de 2013.

\section{BIBLIOGRAFÍA}

AGAUR (2011). CARHUS Plus+ 2010. http:// www10.gencat.cat/agaur_web/AppJava/a_info. jsp?contingut=carhus_FAQ_2010\#id_1 [Consulta 10 de febrero de 2013].

Archambault, E., y Larivière, V. (2010). The limits of bibliometrics for the analysis of the social sciences and humanities literature. En: International Social Science Council, World Social Sciences Report: Knowledge Divides, Paris: UNESCO.

Ardanuy, J.; Urbano, C.; Quintana, L. (2009). A citation analysis of Catalan literary studies (1974-2003): Towards a bibliometrics of humanities studies in minority languages. Scientometrics 81 (2), 347-366. http://dx.doi.org/10.1007/s11192-008-2143-3

Besselaar, P. van den; Edler, J.; Heimeriks, G.; Henriques, L.; Laredo, P.; Luukkonen, T.; Nedeva, M.; Schoen, A. y Thomas, D. (2007). Toward ERA configurations. An experiment on Chemistry, Worshop «Beyond the dichotomy of national vs. European science systems. Configuration of knowledge, institutions and policy in European research», Bonn, 30 May.

Bonaccorsi, A.; Daraio, C.; Lepori, B. y Slipersaeter, S. (2007). Indicators for the analysis of Higher Education Systems: some methodological reflections. Research Evaluation, 16 (2), 66-78. http://dx.doi.org/10.3152/095820207X218141

Burgueño Rivero, J. (1997). Geografia lingüística d'Espanya. Treballs de la Societat Catalana de Geografia, 43 (12), 35-57.

Cañibano, C. y Bozeman, B. (2009). Curriculum vitae method in science policy and research evaluation: the state of the art. Research Evaluation, 18 (2), 8694. http://dx.doi.org/10.3152/095820209X441754

Coneixements $i$ usos del català a Catalunya el 2010: dades del Baròmetre de la Comunicació i la Cultura (2011). Institut d'Estudis Catalans \& Acadèmia Catalana de les Ciències i les Humanitats. http:// www.fundacc.org/docroot/fundacc/pdf/dieta_ llengua.pdf [Consulta 10 de febrero de 2013].

Filippo, D. de.; Sanz-Casado, E.; Urbano, C.; Ardanuy, J y Gómez, I. (2011). El papel de las bases de datos institucionales en el análisis de la actividad científica de las universidades. Revista Española de Documentación Científica vol. 34 (2), 165-189. http://dx.doi.org/10.3989/redc.2011.2.797

Moya, F. de (dir.) (2013). Indicadores Bibliométricos de la Actividad Científica Española 2010. Madrid: Fundación Española para la Ciencia y la Tecnología.

Gantman, R. (2011). La productividad científica argentina en Ciencias Sociales: Economía, Psicología, Sociología y Ciencia Política en el CONICET (2004-2008). Revista Española de Documentación Científica vol. 34 (3), 408-425. http://dx.doi.org/10.3989/redc.2011.3.829

Georgas, H. y Cullars, J. (2005). A citation study of the characteristics of the lingüístics Literature, College and research libraries, 66 (6), 496-515.

Gomez, I.; Bordons, M.; Fernández, M. T. y Morillo, F. (2009). Structure and Research Performance of Spanish Universities. Scientometrics, 79 (1), 131146. http://dx.doi.org/10.1007/s11192-009-0408-0 
Hicks, D. (2004). The Four Literatures of Social Science. En: Moed, H., Glänzel, W. y Schmoch, U. (eds.), Handbook of Quantitative Science and Technology Research. The Use of Publication and Patent Statistics in Studies of S\&T Systems, Springer, New York, pp. 473-96.

Iribarren-Maestro, I. ; Lascurain-Sánchez, M. L., y SanzCasado, E. (2009). The use of bibliometric techniques in evaluating Social Sciences and Humanities. En: Åström, F.; Danell, R.; Larsen, B.; Schneider, J. W. Celebrating Scholarly Communication Studies: A Festschrift for Olle Persson at his 60th Birthday, pp. 25-37. Eds. International Society for Scientometrics and Informetrics.

Larédo, Ph. (2003). University Research Activities: Ongoing Transformations and New Challenges. Higher Education Management and Policy, 15 (1), 105-123. http://dx.doi.org/10.1787/hemp-v15-art8-en

Lepori, B.; Barré, R., y Filliatreau, Gh. (2008). New Perspectives and Challenges for the Design of S\&T Indicators. Research Evaluation, 17, 33-44. http:// dx.doi.org/10.3152/095820208X291176

Moed, H.F. (2005), Citation Analysis in Research Evaluation, Springer, Berlin.

Nederhof, A. J. y Noyons, E. C. M. (1992). International comparison of departments' research performance in the humanities, Journal of the American Society for Information Science and Technology, 43(3), 249-256. http://dx.doi.org/10.1002/(SICI)10974571(199204)43:3<249:: AID-ASI7>3.0.CO;2-I

Nederhof, A. J. (2006). Bibliometric monitoring of research performance in the Social Sciences and the Humanities: a review. Scientometrics, 66(1), 81100. http://dx.doi.org/10.1007/s11192-006-0007-2
Osca Lluch, J. y Mateo Marquina, M. E. (2003). Difusión de las revistas españolas de ciencias sociales y humanidades. Acercamiento bibliométrico. Revista General de Información y Documentación, 13 (1), 115-132.

Raan, A. van (2004). Measuring Science. En: Moed, H., Glänzel, W. y Schmoch U. (eds.), Handbook of Quantitative Science and Technology Research, Dordrecht: Kluwer Academic Publishers, pp. 19-50.

Rovira, L. (2006). Presentació del projecte CARHUS, classificació de revistes científiques en ciències socials i humanitats. Treballs de comunicació, 21, 45-50.

Soriano, J. (2008). El efecto ANECA. Congreso internacional fundacional de la AE-IC. Santiago de Compostela. Disponible en: http://www.aeic.org/ santiago2008/contents/pdf/comunicaciones/286. pdf. [Consulta 10 de febrero de 2013].

Torres-Salinas, D. L. y Moed, H. F. (2009). Library Catalog. Analysis as a tool in studies of Social Sciences and Humanities: An exploratory study of published book titles in Economics. Journal of Informetrics, 3 (1), 9-26. http://dx.doi. org/10.1016/j.joi.2008.10.002

Torres-Salinas, D. L.; Bordons, M.; Giménez-Toledo, E.; Delgado-López-Cózar, E.; Jiménez-Contreras, E. y Sanz-Casado, E. (2010). Clasificación integrada de revistas científicas (CIRC): propuesta de categorización de las revistas en ciencias sociales y humanas. El profesional de la información, 19 (6), 675-683. http://dx.doi.org/10.3145/epi.2010.nov.15

Zwaan, R. A y Nederhof, A. J. (1990). Some aspects of scholarly communication in linguistics: An empirical study, Language, 66, 523-527. http://dx.doi. org/10.2307/414612 